\title{
Optimisation of Constrained Multi-layer Absorbers by Using Genetic Algorithms
}

\author{
Ying-Chun Chang, Long-Jyi Yeh and Min-Chie Chiu \\ Department of Mechanical Engineering, Tatung University, Taipei, Taiwan 104, R.O.C.
}

(Received 3 November 2003; accepted 24 June 2004)

\begin{abstract}
For maintenance and access purposes, it is necessary to minimise the thickness of a sound absorption control system. In this paper, the maximal sound absorption under space constraints is studied. The genetic algorithm (GA) is adopted as the optimiser, and the GA searching technique is presented. Case studies of optimisations on normal incidence sound absorption with respect to three kinds of sound absorbers (single-layer, double-layer, and triple-layer) at the pure tone of $350 \mathrm{~Hz}$ under a fixed thickness are introduced. The optimised parameters include 1) absorbing material; 2) diameters of holes on the perforated plates; 3 ) perforated ratios of the perforated plates; and 4) the flow resistivities of the absorbing materials. Before optimisation, the accuracy of a single-layer absorber mathematical model is tested. Thereafter, the optimisations of three kinds of sound absorbers with one to three layers are exemplified. Consequently, results reveal that the sound absorption at the desired frequency is almost maximised. The more layers there are in the sound absorber, the higher the broadband. The optimal design of a multi-layer sound absorbers system proposed in this study can provide a quick and efficient approach (without redundant testing).
\end{abstract}

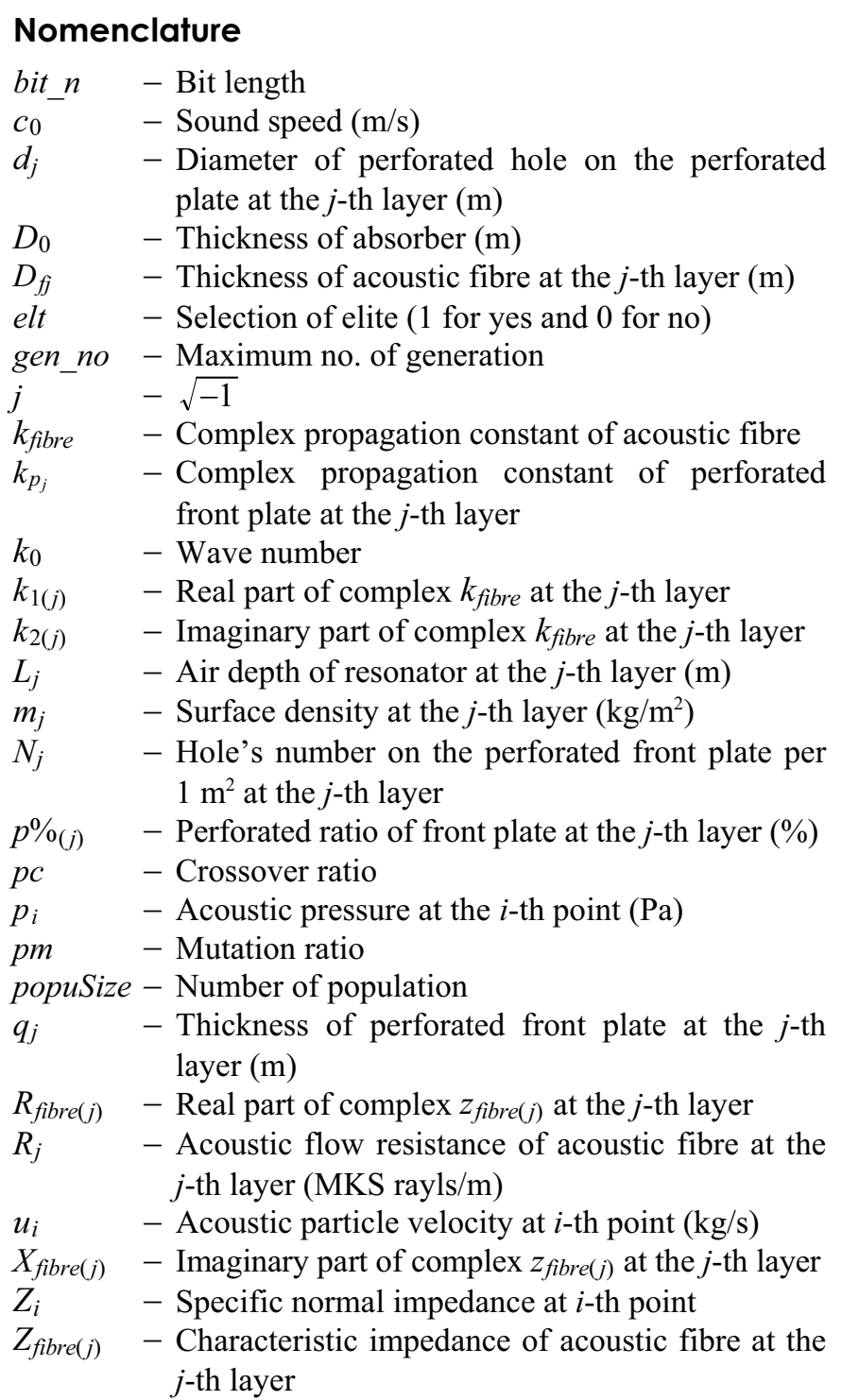

$Z_{p i} \quad$ - Characteristic impedance of perforated front plate at $i$-th point

$\omega \quad$ - Angular frequency $(\mathrm{rad} / \mathrm{s})$

a - Sound absorption coefficient of absorber

$\rho_{0} \quad-$ Air density $\left(\mathrm{kg} / \mathrm{m}^{3}\right)$

\section{INTRODUCTION}

Even though there is a proliferation of studies on sound absorbers, a discussion of the optimal design of sound absorbers (under space constraints) is rarely emphasised. A graphic analysis of sound absorption on perforated singlelayer absorbers was performed and discussed in the previous work. ${ }^{1}$

In order to efficiently finalise the optimal design of a multilayer absorber system, a series of case studies on optimisation under thickness constraints, as well as the new optimiser of a genetic algorithm, was introduced.

Recently, genetic algorithms have been successfully applied to many disciplines. GA optimisers are robust, stochastic search methods modelled on the concepts of natural selection and evolution. ${ }^{2,3}$ Unlike the traditional gradient method, which needs the derivatives and a good starting point in the objective function, GA optimisers are able to locate the global optimum in a near optimal manner easily. In this paper, a matrix transfer conception for sound absorption (applied in the derivation of a normal sound absorption coefficient) was coupled with the searching technique of the genetic algorithm. In addition, the semi-empirical formulas of specific normal impedance by Delany and Bazley, as well as those by Bolt ${ }^{5}$ and Bolt and Ingard ${ }^{6}$, are included in the model derivation.

\section{THEORETICAL BACKGROUND}

This paper considers a multi-layer sound absorber with partitions, shown in Fig. 1. Three kinds of sound absorbers 